\title{
Response to the discussion of "The effect of parameter uncertainty on achieved safety integrity of safety system"
}

\author{
Ming $\mathrm{Xu}^{1}$, Tao Chen ${ }^{2, *}$, Xianhui Yang ${ }^{1}$ \\ ${ }^{1}$ Department of Automation, Tsinghua University, Beijing 100084, China \\ ${ }^{2}$ Division of Civil, Chemical and Environmental Engineering, University of Surrey, Guildford \\ GU2 7XH, UK \\ ${ }^{*}$ Corresponding author. Tel.: +44 1483 686593; Fax: +44 1483686581 \\ Email: t.chen@surrey.ac.uk
}

This letter is in response to the discussion of our recent paper entitled "The effect of parameter uncertainty on achieved safety integrity of safety system" [1]. We are grateful to the discussants for their constructive comments and suggestions, and in particular their insight into our proposed methods to measure safety-related (SR) uncertainty. The detailed response is given below.

We agree that the second index, $M 1_{i}^{\prime}$, is always zero for any input parameter. Thus, this index is indeed incapable of assessing the parameters' importance. In all four examples in the original paper [1], $S_{Y} \approx E\left(S_{Y \mid X_{i}}\right)$ was empirically observed subject to numerical errors. In this regard, we thank the discussants for providing a theoretical proof of this fact.

Combining the idea of the original paper [1] and Borgonovo's moment-independent importance measure index $\delta_{i}$ [2], the discussants suggested the following index for measuring the mean effect of parameter uncertainty on SR uncertainty:

$$
M_{i}=\int_{-\infty}^{+\infty} f_{X_{i}}\left(x_{i}\right)\left[\int_{p_{S L L x}}^{+\infty}\left|f_{Y}(y)-f_{Y \mid X_{i}}(y)\right| d y\right] d x_{i}
$$

which, unfortunately, is not suitable for measuring the SR uncertainty as defined in [1]. Fig. 1 illustrates an example where parameter $X_{i}$ is considered. Assume $X_{i}$ takes two values, $x_{1}$ and $x_{2}$, with probabilities $p$ and $1-p(0 \leqslant p \leqslant 1)$, respectively. Eq. (1) calculates the expected 
difference between $f_{Y}\left(y \geq p_{S I L x}\right)$ and $f_{Y \mid X_{i}=x_{j}^{*}}\left(y \geq p_{S I L x}\right)$ with respect to $x_{j}^{*}, j=1,2$ (shaded area in Fig. 1(a) and (c)), while the SR uncertainty concerns with the change of unsafety probability (equivalently the unsafety region). As shown in Fig. 1 (b) and (d), given $X_{i}=x_{j}^{*}$, the size of conditional unsafety region $S_{Y \mid X_{i}=x_{j}^{*}}$ is the same as that of original unsafety region $S_{Y \mid X_{i}}$, and thus the parameter $X_{i}$ has no contribution to SR uncertainty. However, the index defined in Eq. (1) gives a nonzero value, i.e. the parameter $X_{i}$ has some contribution to the uncertainty. Cleary, this is not consistent with the concept of SR uncertainty. Although this is a simplified example with a discrete random variable $X_{i}$, it highlights that Eq. (1) calculates the change in probability density function, while SR uncertainty is focused on the change in size of unsafety region. Therefore, Eq. (1) is not recommended for measuring the impact of parameter uncertainty on SR uncertainty.

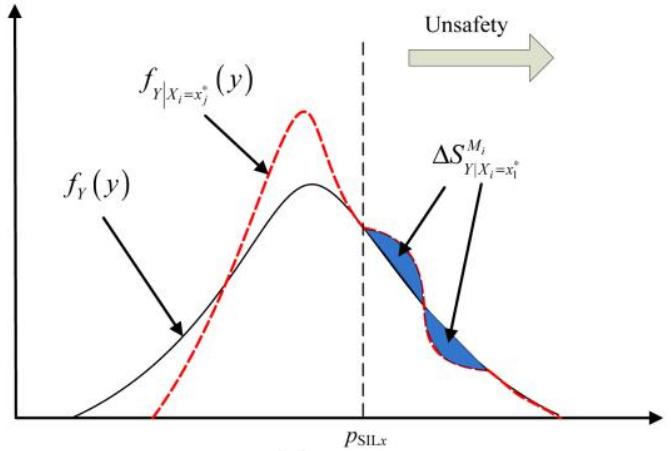

(a)

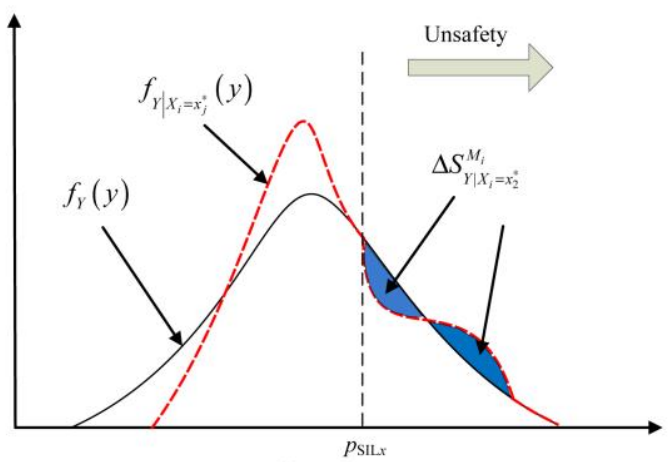

(c)

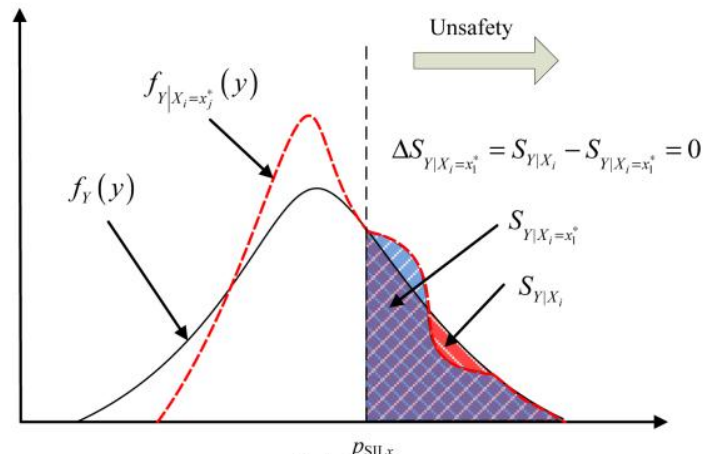

(b)

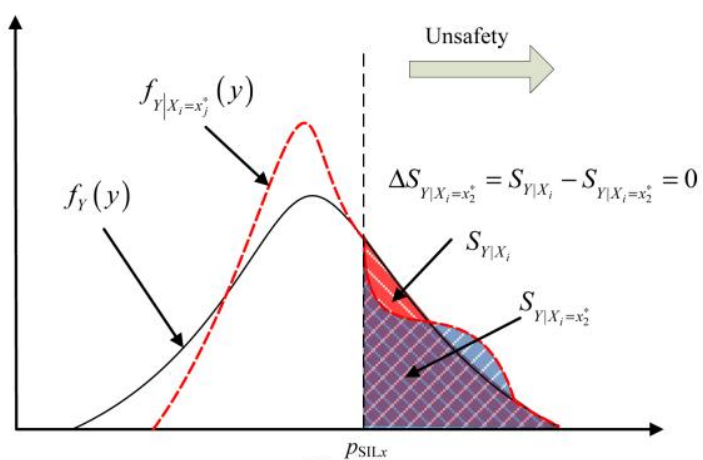

(d)

Fig. 1. The density $f_{Y}(y)$ (solid) and conditional density $f_{Y \mid X_{i}=x_{j}^{*}}(y)$ (dashed). 
Finally, the discussants suggested that the third index defined in [1] is the same as the reliability sensitivity index proposed by $\mathrm{Wu}[3,4]$. They are indeed similar subject to minor difference in the denominator (standard deviation in Wu's index and variance in ours). More importantly, this index was used in $[3,4]$, and other situations, with no reference to the concept of SR uncertainty, which was the focus of our work.

In summary, we still recommend to measure the SR uncertainty by using $\mathrm{M} 1_{i}, \mathrm{M} 2{ }_{i}$ and $\mathrm{M}_{i}$. We sincerely thank the discussants for their effort in presenting a constructive criticism of our work. It is hoped that our contribution and the discussion will initiate further interest in SR uncertainty in the safety community.

\section{References}

[1] Xu M, Chen T, Yang XH. The effect of parameter uncertainty on achieved safety integrity of safety system. Reliability Engineering \& System Safety, 2012, 99: 15-23.

[2] Borgonovo E. A new uncertainty importance measure. Reliability Engineering \& System Safety, 2007, 92: 771-784.

[3] $\mathrm{Wu} \mathrm{YT}$. Computational methods for efficient structural reliability and reliability sensitivity analysis. AIAA Journal, 1994, 32: 1717-1723.

[4] Wu YT, Mohanty S. Variable screening and ranking using sampling-based sensitivity measures. Reliability Engineering \& System Safety, 2006, 91: 634-647. 